\title{
On the Lebesgue Property of Monotone Convex Functions
}

\author{
Keita Owari \\ Graduate School of Economics, The University of Tokyo \\ 7-3-1 Hongo, Bunkyo-ku, Tokyo 113-0033, Japan \\ owari@e.u-tokyo.ac.jp
}

ABSTRACT

\author{
INFO \\ MSC 2010: 46E30, 47H07, 46N10, 91G80, 91B30 \\ JEL: C02, C60 \\ First Version: 4 Jun. 2013, Accepted: 16 Nov. 2013 \\ To appear in: Math. Financ. Econ. \\ DOI: $10.1007 / \mathrm{s} 11579-013-0111-z$
}

The Lebesgue property (order-continuity) of a monotone convex function on a solid vector space of measurable functions is characterized in terms of (1) the weak inf-compactness of the conjugate function on the order-continuous dual space, (2) the attainment of the supremum in the dual representation by order-continuous linear functionals. This generalizes and unifies several recent results obtained in the context of convex risk measures.

Key Words: monotone convex functions, Lebesgue property, order-continuity, perturbed James's theorem, convex risk measures

\section{Introduction and the Statement of the Result}

Many problems in mathematical finance and economics involve some monotone convex functions of measurable functions, and their regularity with respect to the natural order structure often plays a key role. In this short note, we characterize the Lebesgue property (order-continuity) of such functions on solid spaces of measurable functions, in terms of the conjugate defined on the order-continuous dual space, which unifies the recent studies in the context of convex risk measures ([6,7], [10], [15], [12]).

We use the probabilistic notation. Throughout the paper, $(\Omega, \mathcal{F}, \mathbb{P})$ denotes a fixed probability space. $L^{0}:=L^{0}(\Omega, \mathcal{F}, \mathbb{P})$ stands for the space of (equivalence classes modulo $\mathbb{P}$-almost sure (a.s.) equality of) finite measurable functions, and we write simply $L^{p}:=L^{p}(\Omega, \mathcal{F}, \mathbb{P})$ for the measure $\mathbb{P}$, while $L^{p}(\mathbb{Q}):=L^{p}(\Omega, \mathcal{F}, \mathbb{Q})$ for other measures. With the a.s. pointwise order, $L^{0}$ is an order-complete Riesz space with the countable-sup property (see [1, Ch. 8] for these terminologies). We fix a solid vector subspace (ideal) $\mathscr{X}$ of $L^{0}$, that is, a vector subspace of $L^{0}$ such that $|X| \leq|Y|$ (a.s.) and $Y \in \mathscr{X}$ imply $X \in \mathscr{X}$. Then $\mathscr{X}$ is an order-complete Riesz space with the countable-sup property on its own right. All Orlicz spaces and their Morse subspaces including $L^{p}$ 's are solid in this sense. We suppose that $\mathscr{X}$ contains the constants, then $L^{\infty} \subset \mathscr{X}$ by the solidness.

We work with the pairing $\left\langle\mathscr{X}, \mathscr{X}_{n}^{\sim}\right\rangle$ where $\mathscr{X}_{n}^{\sim}$ is another solid space given by

$$
\mathscr{X}_{n}^{\sim}=\left\{Z \in L^{0}: X Z \in L^{1}, \forall X \in \mathscr{X}\right\},
$$

with the bilinear form $\langle X, Z\rangle=\mathbb{E}[X Z]:=\int_{\Omega} X Z d \mathbb{P}$. In the terminology of Riesz spaces, $\mathscr{X}_{n}^{\sim}$ is the order-continuous dual of $\mathscr{X}$ (with the identification of $Z \in \mathscr{X}_{n}^{\sim}$ and the order-continuous linear functional $X \mapsto \mathbb{E}[X Z]$ on $\mathscr{X}$, see [19, Section 112]). Note that $\mathscr{X}$ separates $\mathscr{X}_{n}^{\sim}$ as long as $\mathscr{X}$ contains the constants as assumed so and then $\mathscr{X}_{n}^{\sim} \subset L^{1}$, while $\mathscr{X}_{n}^{\sim}=\{0\}$ is possible in general. $\mathscr{X}_{n}^{\sim}$ separates $\mathscr{X}$ if and only if $\mathscr{X} \subset L^{1}(\mathbb{Q})$ for a probability measure $\mathbb{Q}$ equivalent to $\mathbb{P}(\mathbb{Q} \sim \mathbb{P})$. The pair $\left\langle\mathscr{X}, \mathscr{X}_{n}^{\sim}\right\rangle$ is then in separating duality, thus the weak topology $\sigma\left(\mathscr{X}, \mathscr{X}_{n}^{\sim}\right)$ is a locally convex Hausdorff topology. 
By a monotone convex function on a solid space $\mathscr{X} \subset L^{0}$, we mean a proper convex function $\varphi: \mathscr{X} \rightarrow(-\infty, \infty]$ such that $\varphi(X) \leq \varphi(Y)$ whenever $X \leq Y$ (a.s.), and let

$$
\varphi^{*}(Z):=\sup _{X \in \mathscr{X}}(\mathbb{E}[X Z]-\varphi(X)), Z \in \mathscr{X}_{n}^{\sim} .
$$

The aim of this note is to prove the following (cf. [6, 7], [10], [15], [12]).

Theorem 1.1. Let $\mathscr{X} \subset L^{0}$ be a solid space containing the constants and contained in $L^{1}(\mathbb{Q})$ for some probability $\mathbb{Q} \sim \mathbb{P}$, and $\varphi: \mathscr{X} \rightarrow \mathbb{R}$ a finite-valued monotone convex function which is $\sigma\left(\mathscr{X}, \mathscr{X}_{n}^{\sim}\right)$-lower semicontinuous or equivalently

$$
\varphi(X)=\sup _{Z \in \mathscr{X}_{n}^{\sim}}\left(\mathbb{E}[X Z]-\varphi^{*}(Z)\right), \forall X \in \mathscr{X} .
$$

Then the following are equivalent:

(1) $\varphi$ has the Lebesgue property on $\mathscr{X}$, that is,

$$
\exists Y \in \mathscr{X} \text { such that }\left|X_{n}\right| \leq|Y|, \forall n \text { and } X_{n} \rightarrow X \text { a.s. } \Rightarrow \varphi(X)=\lim _{n} \varphi\left(X_{n}\right) ;
$$

(2) $\varphi^{*}$ is $\sigma\left(\mathscr{X}_{n}^{\sim}, \mathscr{X}\right)$-inf-compact, that is, $\left\{Z \in \mathscr{X}_{n}^{\sim}: \varphi^{*}(Z) \leq c\right\}$ is $\sigma\left(\mathscr{X}_{n}^{\sim}, \mathscr{X}\right)$-compact for each $c>0$

(3) $\sup _{Z \in \mathscr{X}_{n}^{\sim}}\left(\mathbb{E}[X Z]-\varphi^{*}(Z)\right)$ is attained for all $X \in \mathscr{X}$ and in particular,

$$
\varphi(X)=\max _{Z \in \mathscr{X}_{n}^{\tilde{n}}}\left(\mathbb{E}[X Z]-\varphi^{*}(Z)\right), \forall X \in \mathscr{X} .
$$

Remark 1.2 (Order-continuity). By the countable-sup property of $\mathscr{X}$ as an ideal of $L^{0}$, the Lebesgue property (1.4) is equivalent to the generally stronger order-continuity: $\varphi(X)=$ $\lim _{\alpha} \varphi\left(X_{\alpha}\right)$ if a net $\left(X_{\alpha}\right)_{\alpha} \subset \mathscr{X}$ converges in order to $X$, i.e., if there is a decreasing net (with the same index set) $\left(\xi_{\alpha}\right)_{\alpha}$ with $\left|X-X_{\alpha}\right| \leq \xi_{\alpha} \downarrow 0$ in $\mathscr{X}$.

A proof will be given in Section 2. Here we collect some remarks and consequences. We first emphasize that all Orlicz spaces as well as their Morse subspaces including $L^{p}$ with $1 \leq p \leq \infty$ are covered by Theorem 1.1. Also, any solid space $\mathscr{X} \subset L^{0}$ which admits a finite monotone convex function is contained in $L^{1}(\mathbb{Q})$ with some $\mathbb{Q} \ll \mathbb{P}$, thus only the equivalence $\mathbb{Q} \sim \mathbb{P}$ does really matter in the assumption regarding $\mathbb{Q}$.

We can relate the Lebesgue property to some other common regularity properties:

Corollary 1.3. In the situation of Theorem 1.1, the equivalent conditions (1) - (3) are further equivalent to any of the following:

(4) $\varphi$ is $\sigma\left(\mathscr{X}, \mathscr{X}_{n}^{\sim}\right)$-subdifferentiable, i.e.,

$$
\forall X \in \mathscr{X}, \exists Z \in \mathscr{X}_{n}^{\sim} \text { such that } \mathbb{E}[X Z]-\varphi(X) \geq \mathbb{E}[Y Z]-\varphi(Y), \forall Y \in \mathscr{X}
$$

(5) $\varphi$ is continuous for the Mackey topology $\tau\left(\mathscr{X}, \mathscr{X}_{n}^{\sim}\right)$.

Proof. (3) $\Leftrightarrow$ (4) is just a paraphrasing since $\bar{Z} \in \mathscr{X}_{n}^{\sim}$ maximizes $Z \mapsto \mathbb{E}[X Z]-\varphi^{*}(Z)$ on $\mathscr{X}_{n}^{\sim}$ if and only if $\mathbb{E}[X \bar{Z}]-\varphi(X)=\varphi^{*}\left(Z_{X}\right)=\sup _{Y \in \mathscr{X}}(\mathbb{E}[Y \bar{Z}]-\varphi(Y))$ by the definition of $\varphi^{*}$. Also, (2) $\Leftrightarrow(5)$ is true for any finite convex function on a vector space forming a dual pair with another vector space, which is lower semicontinuous w.r.t. a topology consistent with the duality (e.g. [13], Propositions 1 and 2). 
Remark 1.4 (On subdifferentiability). The message of (4) is that the subdifferential of $\varphi$ contains a $\sigma$-additive element (rather than it is non-empty). Consider the case where $\mathscr{X}$ is given a completely metrizable topology $\tau$ for which $(\mathscr{X}, \tau)$ is a locally convex Fréchet lattice (w.r.t. the same a.s. pointwise order). Then the $\tau$-dual of $\mathscr{X}$ has the direct sum decomposition $\mathscr{X}^{*}=\mathscr{X}_{n}^{\sim} \oplus \mathscr{X}_{s}^{\sim}$ where $\mathscr{X}_{s}^{\sim}$ is the set of singular linear functionals on $\mathscr{X}$ (see $[1$, Ch. 8] for detail). In this case, an extended Namioka-Klee theorem of [3] asserts that any finite monotone convex function $\varphi$ on $\mathscr{X}$ is automatically $\tau$-continuous and subdifferentiable in the usual sense: for any $X \in \mathscr{X}$, there exists a $v \in \mathscr{X}^{*}$ such that

$$
v(X)-\varphi(X) \geq v(Y)-\varphi(Y), \forall Y \in \mathscr{X} .
$$

See [3, Theorem 1] (see also [18, Proposition 3.1]). Thus when $\mathscr{X}$ is a locally convex Fréchet lattice, Corollary 1.3 tells us that such $v$ can be taken to be order continuous $(\Leftrightarrow \sigma$-additive in our setting) if and only if $\varphi$ has the Lebesgue property (the sufficiency is already obtained by [3, Lemma 7]).

As the Lebesgue property (1.4) is the continuity w.r.t. the order structure, the order lower semicontinuity is often called the Fatou property and characterized by

$$
\exists Y \in \mathscr{X} \text { such that }\left|X_{n}\right| \leq|Y|, \forall n \text { and } X_{n} \rightarrow X \text { a.s. } \Rightarrow \varphi(X) \leq \liminf _{n} \varphi\left(X_{n}\right) .
$$

Clearly, (1.4) implies (1.8), and the latter is closely related to the $\sigma\left(\mathscr{X}, \mathscr{X}_{n}^{\sim}\right)$-lower semicontinuity $(\Leftrightarrow(1.3)$ ) assumed in Theorem 1.1. In fact, $(1.3) \Rightarrow(1.8)$ is always true (see [3, Proposition 1]). If the converse was also true, Theorem 1.1 would provide us an even nicer interpretation of the Lebesgue property as the Fatou property (easy to check) plus "something extra", with the "extra" being precisely specified. See [16, Theorem 3.9] for more discussion, where a characterization of Lebesgue property in the form of Theorem 1.1 is obtained under solely the Fatou property as the a priori assumption, but with the conjugate $\left(\left.\varphi\right|_{L^{\infty}}\right)^{*}$ of the restriction to $L^{\infty}$ of $\varphi$ instead of $\varphi^{*}$. The implication (1.8) $\Rightarrow(1.3)$ for proper convex functions is indeed true for some good spaces $\mathscr{X}$, but its validity in the generality of this paper is still open (to us).

Remark 1.5. The above question regarding the Fatou and $\sigma\left(\mathscr{X}, \mathscr{X}_{n}^{\sim}\right)$-lower semicontinuity is equivalent to asking if all order closed convex sets in $\mathscr{X}$ are $\sigma\left(\mathscr{X}, \mathscr{X}_{n}^{\sim}\right)$-closed. In [3, Lemma 6 and Corollary 4], it is claimed that this is true whenever $\mathscr{X}$ is (lattice homomorphic to) an ideal of $L^{1}$, but the proof given there has an error. Adapting to our notation, they argued that as $\mathscr{X}$ being an ideal of $L^{1}, \mathscr{X}_{n}^{\sim}$ contains $L^{\infty}$, so the $\sigma\left(\mathscr{X}, \mathscr{X}_{n}^{\sim}\right)$-convergence of a net $\left(X_{\alpha}\right)_{\alpha}$ in $\mathscr{X}$ to $X$ implies the $\sigma\left(L^{1}, L^{\infty}\right)$-convergence to the same limit as a net in $L^{1}$, and consequently there exist a sequence of indices $\left(\alpha_{n}\right)_{n}$ as well as $Y_{n} \in \operatorname{conv}\left(X_{\alpha_{n}}, X_{\alpha_{n+1}}, \ldots\right)$ such that $Y_{n} \rightarrow X$ in order in $L^{1}$. From this they concluded that $Y_{n} \rightarrow X$ in order in $\mathscr{X}$, which would prove the desired implication for all convex sets in $\mathscr{X}$. The error lies in the last part. More specifically, the order convergence of a sequence in an ideal $\mathscr{X}$ of $L^{0}$ is equivalent to the dominated a.s. convergence (i.e., $X_{n} \rightarrow X$ a.s. and $\exists Z \in \mathscr{X}_{+}$with $\left|X_{n}\right| \leq Z$ a.s. for all $n$ ); the a.s. convergence is common to all ideals of $L^{0}$, while being dominated by an element of $\mathscr{X}$ is specific to each $\mathscr{X}$, so $Y_{n} \rightarrow X$ in order as a sequence in $L^{1}$ need not imply the order convergence in $\mathscr{X}$.

\subsection{Examples and Related Literature}

A monotone decreasing convex function $\rho: \mathscr{X} \rightarrow(-\infty, \infty]$ is called a convex risk measure if it satisfies $\rho(X+c)=\rho(X)-c$ for all constants $c$ (see [9] for financial motivation and use of this 
notion). Making a change of sign, $\varphi(X)=\rho(-X)$ is a monotone (increasing) convex function with

$$
\varphi(X+c)=\varphi(X)+c, X \in \mathscr{X}, c \in \mathbb{R} .
$$

For this type functions, which we call convex risk functions, [12, Theorem 4.1] obtained a similar result regarding the equivalence between (1) and (3), and the characterization in the form of Theorem 1.1 including the weak compactness (2) has been studied for some special solid spaces $\mathscr{X}$ as briefly reviewed below.

\subsection{1 $L^{\infty}$}

When $\mathscr{X}=L^{\infty}$, then $\left(L^{\infty}\right)_{n}^{\sim}=L^{1}$, thus $\sigma\left(L^{\infty},\left(L^{\infty}\right)_{n}^{\sim}\right)=\sigma\left(L^{\infty}, L^{1}\right)$ is the weak* topology. Then on the one hand, a convex set $C \subset L^{\infty}$ is $\sigma\left(L^{\infty}, L^{1}\right)$-closed if and only if $\left\{X \in C:\|X\|_{\infty} \leq a\right\}$ is $L^{0}$-closed for all $a>0$ by the Krein-Šmulian theorem, and on the other hand, the Fatou property (1.8) is equivalent to

$$
\sup _{n}\left\|X_{n}\right\|_{\infty}<\infty, X_{n} \rightarrow X \text { a.s. } \Rightarrow \varphi(X) \leq \liminf _{n} \varphi\left(X_{n}\right) .
$$

Consequently, we have (1.3) $\Leftrightarrow(1.8)$.

In the case of $L^{\infty}$, the equivalence of (1) - (3) is first obtained by [6] for sublinear expectation (or equivalently coherent risk measures), i.e., for positively homogeneous monotone convex functions $\varphi$ with (1.9). The case of convex risk functions is then proved by [10] with an additional assumption that $L^{1}$ is separable, and the latter assumption is later removed by [7] by a homogenization trick. As a crucial (but trivial) feature of the space $L^{\infty},(1.9)$ and the monotonicity already imply that $\varphi$ is finite everywhere. Thus Theorem 1.1 slightly generalizes the previous results just mentioned, and this generalization is crucial in the implication (3) $\Rightarrow$ (2) (while not essential for other implication), where a consequence of (1.9) that all the level sets $\left\{Z \in L^{1}: \varphi^{*}(Z) \leq c\right\}$ are bounded in norm was used to invoke (the proof of) James's suptheorem.

Finally, we mention a remarkable fact, due to [8], that the Lebesgue property on $L^{\infty}$ of a convex risk function is necessary for the function to have a finite extension to some solid rearrangement invariant space $\mathscr{X} \subset L^{0}$ properly containing $L^{\infty}$.

\subsubsection{Orlicz Spaces and their Morse Subspaces (Orlicz hearts)}

Let $\Phi:[0, \infty) \rightarrow[0, \infty]$ be a Young function (increasing left-continuous convex function finite on a neighborhood of 0 with $\Phi(0)=0$ and $\lim _{x \rightarrow \infty} \Phi(x)=\infty$ ) and let

$$
\begin{aligned}
& L^{\Phi}:=\left\{X \in L^{0}: \exists \lambda>0, \mathbb{E}[\Phi(\lambda|X|)]<\infty\right\} \quad \text { (Orlicz space), } \\
& M^{\Phi}:=\left\{X \in L^{0}: \forall \lambda>0, \mathbb{E}[\Phi(\lambda|X|)]<\infty\right\} \quad \text { (Morse subspace). }
\end{aligned}
$$

Then both $L^{\Phi}$ and $M^{\Phi}$ are solid spaces, $L^{\infty} \subset L^{\Phi} \subset L^{1}$ and $\left(L^{\Phi}\right)_{n}^{\tilde{n}}=L^{\Phi^{*}}$ in general, where $\Phi^{*}(y):=\sup _{x \geq 0}(x y-\Phi(x))$ is the conjugate Young function.

If $\Phi$ is finite-valued (otherwise $\left.M^{\Phi}=\{0\}\right)$ ), then $M^{\Phi}$ contains $L^{\infty}$ and $\left(M^{\Phi}\right)_{n}^{\sim}=\left(M^{\Phi}\right)^{*}=L^{\Phi^{*}}$ as well. Thus in this case, $\sigma\left(M^{\Phi},\left(M^{\Phi}\right)_{n}^{\sim}\right)=\sigma\left(M^{\Phi}, L^{\Phi^{*}}\right)$ is the weak topology, and consequently (1.3) for $\mathscr{X}=M^{\Phi}$ is equivalent to the norm-lower semicontinuity. Any norm convergent sequence in $M^{\Phi}$ admits a subsequence which is dominated by some element of $M^{\Phi}$ and a.s. convergent to the same limit, which shows the implication $(1.8) \Rightarrow(1.3)$. In fact, the Luxemburg norm $\|\cdot\|_{\Phi}$ (see [17] for definition) is order-continuous on $M^{\Phi}$ (though not on $L^{\Phi}$ ). Consequently, the extended Namioka-Klee theorem tells us that any finite monotone convex function 
$\varphi$ on $M^{\Phi}$ automatically has the Lebesgue property. The equivalence of finiteness and (2), (3) in this case (with (1.9)) is contained in [5] in a slightly different form, where it is also shown that the finiteness of a convex risk function is equivalent to int $\operatorname{dom} \varphi \neq \emptyset$. See also [11] for the case $\mathscr{X}=L^{p}=M^{\Phi_{p}}\left(\Phi(x)=x^{p} / p, 1 \leq p<\infty\right)$.

Recall that the Young function $\Phi$ is said to satisfy the $\Delta_{2}$-condition if there exist constants $C>0$ and $x_{0}>0$ such that $\Phi(2 x) \leq C \Phi(x)$ for $x \geq x_{0}$. In this case, $L^{\Phi}=M^{\Phi}$ (the converse is also true if $(\Omega, \mathcal{F}, \mathbb{P})$ is atomless). Consequently, $\left(L^{\Phi}\right)_{n}^{\sim}=\left(L^{\Phi}\right)^{*}=L^{\Phi^{*}}$, thus the argument of previous paragraph still applies.

When $\mathscr{X}=L^{\Phi}$ with $\Phi^{*}$ finite, [15] recently obtained the same equivalence but under a stronger a priori assumption that $\varphi$ is $\sigma\left(L^{\Phi}, M^{\Phi^{*}}\right)$-lower semicontinuous. Though the latter assumption is not so unreasonable, it excludes some trivial cases unless $L^{\Phi^{*}}=M^{\Phi^{*}}$ : For any $Z \in L_{+}^{\Phi^{*}} \backslash M^{\Phi^{*}}, \varphi(X)=\mathbb{E}[X Z]$ is a monotone convex $\sigma\left(L^{\Phi}, L^{\Phi^{*}}\right)$-lsc function on $L^{\Phi}$, but is not $\sigma\left(L^{\Phi}, M^{\Phi^{*}}\right)$-continuous (thus not lsc by linearity) because $\left(L^{\Phi}, \sigma\left(L^{\Phi}, M^{\Phi^{*}}\right)\right)^{*}=M^{\Phi^{*}}$. Note that this $\varphi$ has the Lebesgue property; if $\left|X_{n}\right| \leq Y \in L^{\Phi}$ and $X_{n} \rightarrow X$ a.s., the dominated convergence theorem applies to the sequence $\left(X_{n} Z\right)_{n}$ dominated by $Y Z \in L^{1}$. Recall that (with $\Phi^{*}$ finite), $\left(M^{\Phi^{*}}\right)^{*}=L^{\Phi}$, hence $\sigma\left(M^{\Phi^{*}}, L^{\Phi}\right)=\sigma\left(M^{\Phi^{*}},\left(M^{\Phi^{*}}\right)^{*}\right)$ (the weak topology). This was needed in [15] to invoke their perturbed James's sup-theorem in the proof of (3) $\Rightarrow$ (2). In fact, our proof of Theorem 1.1 use a similar version of James's theorem but not to $\mathscr{X}_{n}^{\sim}$. We reduce the problem to $\sigma\left(L^{1}, L^{\infty}\right)$ by a simple "change of variable" trick, which allows us to prove the implication for arbitrary solid space $\mathscr{X}$ containing $L^{\infty}$ and contained in $L^{1}(\mathbb{Q})$ with some $\mathbb{Q} \sim \mathbb{P}$ under the weaker assumption (1.3). The equivalence of (1.3) and the Fatou property (1.8) in this level of generality is still open (to us).

\section{Proof of Theorem 1.1}

In the sequel, all the assumptions of Theorem 1.1 are in force without further mentioning. Recall that $\mathscr{X}$ is an order-complete (Dedekind-complete) Riesz space with the countable-sup property as an ideal of $L^{0}$, whose order-continuous dual space is identified with $\mathscr{X}_{n}^{\sim}$ given by (1.1), and we have $\mathscr{X}_{n}^{\sim}=\mathscr{X}_{c}^{\sim}$ (the $\sigma$-order-continuous dual) in the notation of [2] as a consequence of the countable-sup property. We shall use the following characterization of $\sigma\left(\mathscr{X}_{n}^{\sim}, \mathscr{X}\right)$-compact sets.

Lemma 2.1. Let $C \subset \mathscr{X}_{n}^{\sim}$. Then the following are equivalent:

(1) $C$ is $\sigma\left(\mathscr{X}_{n}^{\sim}, \mathscr{X}\right)$-relatively compact,

(2) $X_{n} \downarrow 0$ in $\mathscr{X}$ implies $\sup _{Z \in C} \mathbb{E}\left[X_{n}|Z|\right] \downarrow 0$.

(3) $\rho_{C}(X):=\sup _{Z \in C} \mathbb{E}[|X Z|]$ is order-continuous, i.e., $X_{n} \in Y \in \mathscr{X},\left|X_{n}\right| \leq|Y|$ a.s. for all $n$ and $X_{n} \rightarrow X$ a.s. imply $\rho_{C}(X)=\lim \rho_{C}\left(X_{n}\right)$.

(4) the set $\{X Z: Z \in C\}$ is uniformly integrable for all $X \in \mathscr{X}$.

Proof. Since $\mathscr{X}$ is order-complete, $C \subset \mathscr{X}_{n}^{\sim}=\mathscr{X}_{c}^{\sim}$ is $\sigma\left(\mathscr{X}_{n}^{\sim}, \mathscr{X}\right)$-relatively compact if and only if the convex solid hull of $C$ is relatively compact for the same topology by [2, Corollary 6.29], and the latter is equivalent to (2) by [2, Theorem 6.21]. To prove (2) $\Rightarrow(3)$, let a sequence $\left(X_{n}\right)_{n}$ in $\mathscr{X}$ be dominated by $Y \in \mathscr{X}$ and converge a.s. to $X$. Then $\bar{X}_{n}:=\sup _{l \geq n}\left|X-X_{l}\right| \leq$ $2 Y$, hence $\bar{X}_{n} \in \mathscr{X}, \bar{X}_{n} \downarrow 0$, thus $\left|\rho_{C}(X)-\rho_{C}\left(X_{n}\right)\right| \leq \sup _{Z \in C} \mathbb{E}\left[\bar{X}_{n}|Z|\right] \downarrow 0$. For (3) $\Rightarrow$ (4), pick an arbitrary $X \in \mathscr{X}$. Then for any sequence $\left(A_{n}\right) \subset \mathcal{F}$ with $\mathbb{P}\left(A_{n}\right) \rightarrow 0$, we have $\left|X \mathbb{1}_{A_{n}}\right| \leq|X| \in \mathscr{X}$ and $X \mathbb{1}_{A_{n}} \rightarrow 0$ a.s. Thus (3) shows that $\sup _{Z \in C} \mathbb{E}\left[|X Z| \mathbb{1}_{A_{n}}\right]=\sup _{Z \in C} \mathbb{E}\left[\left|X \mathbb{1}_{A_{n}}\right||Z|\right] \downarrow 0$, hence $\{X Z: Z \in C\}$ is uniformly integrable for all $X \in \mathscr{X}$.

(4) $\Rightarrow(2)$. Take $\mathbb{Q} \sim \mathbb{P}$ such that $\mathscr{X} \subset L^{1}(\mathbb{Q})$ and put $\hat{Z}:=d \mathbb{Q} / d \mathbb{P}$. Then note that $\mathbb{P}(\hat{Z}>$ $0)=1$ and $\sup _{Z \in C} \mathbb{Q}(Z / \hat{Z}>N) \leq \sup _{Z \in C} \mathbb{E}[|Z|] / N \rightarrow 0(N \rightarrow \infty)$ since $C$ is bounded in $L^{1}$ by 
taking $X=1 \in \mathscr{X}$ in (4). Since the one-point set $\{1 / \hat{Z}\}=\{d \mathbb{P} / d \mathbb{Q}\}$ is $\mathbb{Q}$-uniformly integrable, we deduce that

$$
\sup _{Z \in C} \mathbb{P}(Z / \hat{Z}>N)=\sup _{Z \in C} \mathbb{E}_{\mathbb{Q}}\left[(1 / \hat{Z}) \mathbb{1}_{\{Z / \hat{Z}>N\}}\right] \rightarrow 0 .
$$

Now suppose $X_{n} \downarrow 0$ in $\mathscr{X}$. Then $\mathbb{E}\left[X_{n}|Z|\right] \leq \mathbb{E}\left[X_{n}|Z| \mathbb{1}_{\{|Z / \hat{Z}|>N\}}\right]+\mathbb{E}\left[X_{n}|Z| \mathbb{1}_{\{|Z / \hat{Z}| \leq N\}}\right] \leq$ $\mathbb{E}\left[X_{1}|Z| \mathbb{1}_{\{|Z / Z|>N\}}\right]+N \mathbb{E}_{\mathbb{Q}}\left[X_{n}\right]$ for all $Z \in C$, hence

$$
\sup _{Z \in C} \mathbb{E}\left[X_{n}|Z|\right] \leq \sup _{Z \in C} \mathbb{E}\left[X_{1}|Z| \mathbb{1}_{\{|Z / \hat{Z}|>N\}}\right]+N \mathbb{E}_{\mathbb{Q}}\left[X_{n}\right] .
$$

The first term in the RHS tends to 0 by the uniform integrability of $\left\{X_{1} Z: Z \in C\right\}$ and (2.1), while for each $N$ fixed, the second term tends to 0 as $n \rightarrow \infty$ by the dominated convergence theorem since $0 \leq X_{n} \leq X_{1} \in L^{1}(\mathbb{Q})$.

The next preparatory result is a version of James's theorem obtained by [14] which we shall use in the proof of $(3) \Rightarrow(2)$.

Theorem 2.2 ([14], Theorem 2). Let $E$ be a real Banach space and $f: E \rightarrow \mathbb{R} \cup\{+\infty\}$ be a function which is not identically $+\infty$ and is coercive, i.e.,

$$
\lim _{\|x\| \rightarrow \infty} \frac{f(x)}{\|x\|}=+\infty .
$$

Then if the supremum $\sup _{x \in E}\left(\left\langle x, x^{*}\right\rangle-f(x)\right)$ is attained for every $x^{*} \in E^{*}$, the level set $\{x \in E$ : $f(x) \leq c\}$ is relatively weakly compact for each $c \in \mathbb{R}$.

Lemma 2.3. Suppose $\varphi(0)=0$, then for any $\beta \in \mathbb{R}, X \in \mathscr{X}$ and $Z \in \mathscr{X}_{n}^{\sim}$,

$$
\mathbb{E}[X Z]-\varphi^{*}(Z) \geq-\beta \Rightarrow \varphi^{*}(Z) \leq 2 \beta+2 \varphi(2|X|) .
$$

Proof. Since $0=\varphi(0)=\inf _{Z \in \mathscr{X}_{n}^{\sim}} \varphi^{*}(Z)$, there exists, for any $\varepsilon>0$, some $Z_{\varepsilon} \in\left(\mathscr{X}_{n}^{\sim}\right)_{+}$with $\varphi^{*}\left(Z_{\varepsilon}\right)<\varepsilon$ (by the monotonicity of $\left.\varphi, \operatorname{dom} \varphi^{*} \subset\left(\mathscr{X}_{n}^{\sim}\right)_{+}\right)$. Thus

$$
\mathbb{E}[X Z] \leq \mathbb{E}\left[2|X| \frac{Z+Z_{\varepsilon}}{2}\right] \leq \varphi(2|X|)+\frac{\varphi^{*}(Z)+\varphi^{*}\left(Z_{\varepsilon}\right)}{2} \leq \varphi(2|X|)+\frac{\varphi^{*}(Z)}{2}+\varepsilon
$$

for any $\varepsilon$, hence $\mathbb{E}[X Z] \leq \varphi(2|X|)+\varphi^{*}(Z) / 2$. Consequently, $\mathbb{E}[X Z]-\varphi^{*}(Z) \geq-\beta$ implies $\varphi^{*}(Z) \leq$ $\beta+\mathbb{E}[X Z] \leq \beta+\varphi(2|X|)+\varphi^{*}(Z) / 2$. Rearranging the terms, we have $\varphi^{*}(Z) \leq 2 \beta+2 \varphi(2|X|)$.

Proof of Theorem 1.1. We suppose without loss of generality that $\varphi(0)=0$ and we write $\Lambda_{c}:=$ $\left\{Z \in \mathscr{X}_{n}^{\sim}: \varphi^{*}(Z) \leq c\right\}$ throughout this proof. Note that $\Lambda_{c}$ is $\sigma\left(\mathscr{X}_{n}^{\sim}, \mathscr{X}\right)$-closed for each $c>0$ since $\varphi^{*}$ is lower semicontinuous for the same topology. Also, the monotonicity of $\varphi$ implies that $\Lambda_{c} \subset \operatorname{dom} \varphi^{*} \subset L_{+}^{1}$.

$(1) \Rightarrow(2)$. By the above comments, it suffices to show that for each $c>0, \Lambda_{c}$ is $\sigma\left(\mathscr{X}_{n}^{\sim}, \mathscr{X}^{\prime}\right)$ relatively compact, which is equivalent (in view of Lemma 2.1) to saying that $\sup _{Z \in \Lambda_{c}} \mathbb{E}\left[X_{n}|Z|\right]$ $\downarrow 0$ if $\mathscr{X} \ni X_{n} \downarrow 0$. Given such a sequence $\left(X_{n}\right)_{n}$, observe that $\sup _{Z \in \Lambda_{c}} \mathbb{E}\left[X_{n} Z\right] \leq$ $\frac{1}{\lambda} \sup _{Z \in \Lambda_{c}}\left(\varphi\left(\lambda X_{n}\right)+\varphi^{*}(Z)\right) \leq \frac{1}{\lambda} \varphi\left(\lambda X_{n}\right)+\frac{c}{\lambda}$ by Young's inequality. Then the Lebesgue property of $\varphi$ implies that $\varphi\left(\lambda X_{n}\right) \downarrow 0$ for every $\lambda>0$ since $X_{n} \downarrow 0$ (so $\lambda X_{n} \downarrow 0$ ). Hence a diagonal argument yields the desired property.

(2) $\Rightarrow(3)$. Note that $Z \mapsto \mathbb{E}[X Z]-\varphi^{*}(Z)$ is $\sigma\left(\mathscr{X}_{n}^{\sim}, \mathscr{X}\right)$-upper semicontinuous (since $\varphi^{*}$ is lower semicontinuous). Thus, the set $\Gamma_{X}:=\left\{Z \in \mathscr{X}_{n}^{\sim}: \mathbb{E}[X Z]-\varphi^{*}(Z) \geq \varphi(X)-1\right\}$ is $\sigma\left(\mathscr{X}_{n}^{\sim}, \mathscr{X}\right)$-closed, and it is contained in the $\sigma\left(\mathscr{X}_{n}^{\sim}, \mathscr{X}\right)$-compact set $\Lambda_{2-2 \varphi(X)+2 \varphi(2|X|)}$ by 
Lemma 2.3, hence $\Gamma_{X}$ itself is $\sigma\left(\mathscr{X}_{n}^{\sim}, \mathscr{X}\right)$-compact. Now (3) is clear since any upper semicontinuous function on a compact set attains its maximum.

(2) $\Rightarrow(1)$. Let $\left(X_{n}\right)_{n}$ be such that $\left|X_{n}\right| \leq Y \in \mathscr{X}_{+}$and $X_{n} \rightarrow X$ a.s., then $|X| \leq Y$ as well, and $\varphi\left(X_{n}\right), \varphi(X) \geq \varphi(-Y)$ by the monotonicity. Thus given (1.3), only those $Z \in \mathscr{X}_{n}^{\sim}$ with $\mathbb{E}\left[X_{n} Z\right]-\varphi^{*}(Z) \geq \varphi(-Y)-1$ contribute to the supremum $\sup _{Z \in \mathscr{X}_{n}^{\sim}}\left(\mathbb{E}[X Z]-\varphi^{*}(Z)\right)$ (and the same is true for $X$ ). Applying Lemma 2.3 and using the notation of the previous paragraph, any such $Z \in \mathscr{X}_{n}^{\sim}$ is contained in $\Lambda_{c_{Y}}$ where $c_{Y}:=2-2 \varphi(-Y)+2 \varphi(2 Y)$. Consequently, $\varphi\left(X_{n}\right)=\sup _{Z \in \Lambda_{c_{Y}}}\left(\mathbb{E}\left[X_{n} Z\right]-\varphi^{*}(Z)\right), \varphi(X)=\sup _{Z \in \Lambda_{c Y}}\left(\mathbb{E}[X Z]-\varphi^{*}(Z)\right)$, thus applying twice the elementary inequality $\sup _{x \in A} f(x)-\sup _{x \in A} g(x) \leq \sup _{x \in A}(f(x)-g(x))$ [e.g., 4, p. 356, Prop. 12],

$$
\begin{aligned}
\left|\varphi(X)-\varphi\left(X_{n}\right)\right| & \leq \sup _{Z \in \Lambda_{c_{Y}}}\left|\mathbb{E}[X Z]-\varphi^{*}(Z)-\left(\mathbb{E}\left[X_{n} Z\right]-\varphi^{*}(Z)\right)\right| \\
& \leq \sup _{Z \in \Lambda_{c_{Y}}} \mathbb{E}\left[\left|X_{n}-X\right| Z\right] \rightarrow 0 .
\end{aligned}
$$

by the $\sigma\left(\mathscr{X}^{\sim}, \mathscr{X}\right)$-compactness of $\Lambda_{c_{Y}}$ and Lemma 2.1 .

$(3) \Rightarrow(2)$. We shall prove that for any $X \in \mathscr{X}$ and $c>0$, the set $\left\{(1+|X|) Z \in L^{1}\right.$ : $\left.Z \in \mathscr{X}_{n}^{\sim}, \varphi^{*}(Z) \leq c\right\}$ is uniformly integrable, which in view of Lemma 2.1 shows the desired compactness. Let us define a function $g_{X}: L^{1} \rightarrow \mathbb{R} \cup\{+\infty\}$ by

$$
g_{X}(Z):= \begin{cases}\varphi^{*}\left(\frac{Z}{1+|X|}\right) & \text { if } \frac{Z}{1+|X|} \in \mathscr{X}_{n}^{\sim}, \quad \forall Z \in L^{1} . \\ +\infty & \text { otherwise, }\end{cases}
$$

Then for any $c>0, g_{X}(Z) \leq c$ iff $Z=(1+|X|) Z^{\prime}, Z^{\prime} \in \mathscr{X}_{n}^{\sim}$ and $\varphi^{*}\left(Z^{\prime}\right) \leq c$, and since $\mathscr{X}$ is an ideal containing $L^{\infty}, \lambda(1+|X|) \operatorname{sgn}(Z) \in \mathscr{X}$. Hence for any $Z \in \operatorname{dom} g_{X}$,

$$
\begin{aligned}
g_{X}(Z) & \geq \mathbb{E}\left[\frac{\lambda(1+|X|) \operatorname{sgn}(Z)}{1+|X|} Z\right]-\varphi(\lambda(1+|X|) \operatorname{sgn}(Z)) \\
& \geq \lambda\|Z\|_{L^{1}}-\varphi(-\lambda(1+|X|)) .
\end{aligned}
$$

Since $\varphi$ is finite on $\mathscr{X}$, this shows that $g_{X}$ is a coercive function on $L^{1}$.

Let $X \in \mathscr{X}, Y \in L^{\infty}$ and $Z_{X, Y} \in \mathscr{X}_{n}^{\sim}$ be a maximizer in (3) for $Y(1+|X|)$ which belongs to $\mathscr{X}$ by the solidness (since $Y(1+|X|) \leq\|Y\|_{\infty}(1+|X|) \in \mathscr{X}$ ). Then

$$
\begin{aligned}
\varphi(Y(1+|X|)) & =\mathbb{E}\left[Y(1+|X|) Z_{X, Y}\right]-\varphi^{*}\left(Z_{X, Y}\right) \\
& =\mathbb{E}\left[Y(1+|X|) Z_{X, Y}\right]-g_{X}\left((1+|X|) Z_{X, Y}\right) .
\end{aligned}
$$

On the other hand, for $Z^{\prime} \in L^{1}$, if $Z^{\prime} \in \operatorname{dom} g_{X}$,

$$
\mathbb{E}\left[Y Z^{\prime}\right]-g_{X}\left(Z^{\prime}\right)=\mathbb{E}\left[Y(1+|X|) \frac{Z^{\prime}}{1+|X|}\right]-\varphi^{*}\left(\frac{Z^{\prime}}{1+|X|}\right) \leq \varphi((1+|X|) Y),
$$

while if $Z^{\prime} \in L^{1} \backslash \operatorname{dom} g_{X}$, then obviously $\mathbb{E}\left[Y Z^{\prime}\right]-g_{X}\left(Z^{\prime}\right)=-\infty \leq \varphi((1+|X|) Y)$. We have thus shown that the supremum $\sup _{Z \in L^{1}}\left(\mathbb{E}[Y Z]-g_{X}(Z)\right)$ is attained by $(1+|X|) Z_{X, Y}$ for all $Y \in L^{\infty}$. Consequently, Theorem 2.2 shows that $\left\{Z^{\prime} \in L^{1}: g_{X}\left(Z^{\prime}\right) \leq c\right\}=\left\{(1+|X|) Z: Z \in \mathscr{X}_{n}^{\sim}, \varphi^{*}(Z) \leq\right.$ $c\}$ is $\sigma\left(L^{1}, L^{\infty}\right)$-relatively compact, hence uniformly integrable by the Dunford-Pettis theorem. $\square$

\section{Acknowledgements}

The author thanks Sara Biagini for helpful comments on an earlier version of the paper. $\mathrm{He}$ also thanks two anonymous referees for careful reading. The financial support of the Center for Advanced Research in Finance (CARF) at the Graduate School of Economics of the University of Tokyo is gratefully acknowledged. 


\section{References}

[1] Aliprantis, C. D. and K. C. Border (2006): Infinite dimensional analysis: A hitchhiker's guide. Springer, Berlin, 3rd ed.

[2] Aliprantis, C. D. and O. Burkinshaw (2003): Locally solid Riesz spaces with applications to economics, Mathematical Surveys and Monographs, vol. 105. American Mathematical Society, Providence, RI, 2nd ed. xii+344 pp.

[3] Biagini, S. and M. Frittelli (2009): On the extension of the Namioka-Klee theorem and on the Fatou property for risk measures. In: Optimality and risk-modern trends in mathematical finance, Springer, Berlin, pp. 1-28.

[4] Bourbaki, N. (1989): General topology. Chapters 1-4. Elements of Mathematics (Berlin). SpringerVerlag, Berlin. Translated from the French, Reprint of the 1966 edition.

[5] Cheridito, P. and T. Li (2009): Risk measures on Orlicz hearts. Math. Finance 19, 189-214.

[6] Delbaen, F. (2000): Coherent risk measures. Cattedra Galileiana. [Galileo Chair]. Scuola Normale Superiore, Classe di Scienze, Pisa.

[7] Delbaen, F. (2009): Differentiability properties of utility functions. In: Optimality and riskmodern trends in mathematical finance, Springer, Berlin, pp. 39-48.

[8] Delbaen, F. (2009): Risk measures for non-integrable random variables. Math. Finance 19, 329333.

[9] Föllmer, H. and A. Schied (2011): Stochastic finance. Walter de Gruyter \& Co., Berlin, 3rd ed. An introduction in discrete time.

[10] Jouini, E., W. Schachermayer and N. Touzi (2006): Law invariant risk measures have the fatou property. Advances in Mathematical Economics 9, 49-71.

[11] Kaina, M. and L. Rüschendorf (2009): On convex risk measures on $L^{p}$-spaces. Math. Methods Oper. Res. 69, 475-495.

[12] Krätschmer, V. (2007): On $\sigma$-additive robust representation of convex risk measures for unbounded financial positions in the presence of uncertainty about the market model. SFB 649 Discussion Paper 2007-010, Humboldt-Universität zu Berlin.

[13] Moreau, J.-J. (1964): Sur la fonction polaire d'une fonction semi-continue supérieurement. C. $R$. Acad. Sci. Paris 258, 1128-1130.

[14] Orihuela, J. and M. Ruiz Galán (2012): A coercive James's weak compactness theorem and nonlinear variational problems. Nonlinear Anal., Theory Methods Appl., Ser. A, Theory Methods 75, 598-611.

[15] Orihuela, J. and M. Ruiz Galán (2012): Lebesgue property of convex risk measures on Orlicz spaces. Math. Financ. Econ. 6, 15-35.

[16] Owari, K. (2013): Maximum Lebesgue extension of monotone convex functions. Preprint, arXiv:1304.7934.

[17] Rao, M. M. and Z. D. Ren (1991): Theory of Orlicz Spaces. Marcel Dekker, Inc.

[18] Ruszczyński, A. and A. Shapiro (2006): Optimization of convex risk functions. Math. Oper. Res. 31, 433-452.

[19] Zaanen, A. C. (1983): Riesz spaces. II, North-Holland Mathematical Library, vol. 30. NorthHolland Publishing Co., Amsterdam. 